\title{
The effect of vertical throughflow in Rivlin-Ericksen elastico-viscous nanofluid in a non-Darcy porous medium
}

\author{
S. Saini, Y. D. Sharma \\ Department of Mathematics, National Institute of Technology Hamirpur, \\ Hamirpur (H.P.), India-177005 \\ shivani2291993@gmail.com
}

DOI 10.17586/2220-8054-2017-8-5-606-612

\begin{abstract}
The instability of convection in a Rivlin-Ericksen elastico-viscous nanofluid with vertical throughflow is investigated using the linear stability theory. A modified Brinkman model is employed and single-term Galerkin method is used to solve the conservation equations. Nine dominating parameters are extracted from the analysis. Due to the combined effect of vertical throughflow, Brownaian motion, and thermophoresis, the Rayleigh number is reduced by a substantial amount. It is found that through flow delays the convection while other nanofluid parameter enhance the convection. The thermal capacity ratio, kinematics viscoelasticity, and Vadasz number do not govern stationary convection. Using the convective component of nanoparticle flux, the critical wave number is a function of nanofluid parameters as well as throughflow parameter. Major trends are investigated briefly by plotting the graphs.
\end{abstract}

Keywords: heat transfer, porous layer, throughflow.

Received: 4 October 2017.

Revised: 9 October 2017

\section{Introduction}

Rivlin and Erickson [1] were the first to propose the theoretical and rheological model for elastic-viscous fluid. Rudraiah et al. [2] studied the overstable convection of an Oldrayd fluid and they found that effect of elasticity is to elongate the cells. Kim et al. [3] found that elasticity parameters have a destabilizing effect on the onset of convection. The effect of permeability, magnetic field, and rotation on Rivlin-Ericksin elastico-viscous fluid was studied by $[4,5]$ and they found that rotation, and magnetic field diminish the instability, while permeability enhances the instability. Sheu [6,7] examined the linear stability in Visco-Elastic nanofluids and studied the combined effects of Brownian diffusion, thermophoresis diffusion, and viscoelasticity. Viscoelastic fluids are widely used in paper coating, chemical industries, and manufacturing processes.

A nanofluid contains nano-sized particles (diameter less than $100 \mathrm{~nm}$ ) which are suspended in the base fluid. The term, "nanofluid" was first coined, by Choi [8] and Buongiorno [9] to describe the importance of the various transport mechanisms in nanofluids. Using the model given by [9], Nield and Kuznetsov [10,11] analyzed the thermal stability problem and found that nanoparticles have a destabilizing effect.

The effect of throughflow was generally stabilizing, however, for a small amount of throughflow, it shows the destabilizing effect $[12,13]$. Nield [14] found that destabilization occurs when the throughflow is away from impermeable boundaries. In the absence of internal heat generation, throughflow destabilized the system in a very small amount of throughflow at the different boundary conditions (top and bottom layer are not identical) [1]. Barletta et al. [15] studied the effect of vertical throughflow with viscous dissipation. Nield and Kuznetsov [16,17] investigated the effect of vertical throughflow on both the boundaries (isoflux and isothermal). By using the Buongiorno model [16], we studied the effect of vertical throughflow on the onset of nanofluid convection, later it, this model was revised by using more realistic boundary conditions [18-20].

From the above brief review, it is clear that many researchers have carried out extensive studies on the onset of convection in Rivlin-Ericksin elastico-viscous nanofluids recently; however, the effect of vertical throughflow in Rivlin-Ericksin elastico-viscous nanofluid using the new boundary conditions (in which nanoparticle flux is the sum of diffusive, connective, and thermophoretic terms) has not been yet studied. Therefore, in this paper, the effects of viscoelastic parameters, nanofluid parameters, and throughflow parameters are investigated analytically and numerically for Rigid-rigid boundary conditions in a non-Darcy porous media. 


\section{Analysis}

\subsection{Mathematical formulation}

An infinite horizontal Rivlin-Ericksen elastico-viscous nanofluid confined between rigid impermeable boundaries $z=0$ and $z=H$ is considered. The pore sizes are large compared to nanoparticles. We use the modified Brinkman-Darcy model to define the nature of the porous medium and the Oberbeck-Boussinesq approximations are employed. We take temperatures $T_{0}$ and $T_{1}\left(T_{1}>T_{0}\right)$ at the lower and the upper wall. The homogeneity and local thermal equilibrium in the porous medium is also assumed. The governing equations for mass, momentum, energy and nanoparticles for Rivlin-Ericksen elastico-viscous nanofluid in porous medium are written below (details of following equations can be seen in the articles given by $[6,7,11]$ :

$$
\begin{gathered}
\nabla \cdot \mathbf{V}_{\mathbf{D}}=0, \\
\frac{\rho_{f}}{\varepsilon} \frac{d \mathbf{V}_{\mathbf{D}}}{d t}=-\nabla \mathbf{p}-\frac{1}{k}\left(\mu+\mu^{\prime} \frac{\partial}{\partial t}\right) \mathbf{V}_{\mathbf{D}}+\left[\varphi \rho_{p}+(1-\varphi)\left\{\rho\left(1-\beta\left(T-T_{0}\right)\right)\right\}\right] \mathbf{g}, \\
\frac{\partial T}{\partial t}+\frac{(\rho c)_{f}}{(\rho c)_{m}} \mathbf{V}_{\mathbf{D}} \cdot \nabla T=k_{m} \nabla^{2} T+\frac{\varepsilon(\rho c)_{p}}{(\rho c)_{m}}\left(D_{B} \nabla \varphi \cdot \nabla T-\frac{\left(\varphi-\varphi_{0}\right) \mathbf{V}_{\mathbf{D}}}{\varepsilon}+\frac{D_{T}}{T_{1}} \nabla T \cdot \nabla T\right), \\
\frac{\partial \varphi}{\partial t}+\frac{1}{\varepsilon} \mathbf{V}_{\mathbf{D}} \cdot \nabla \varphi=D_{B} \nabla^{2} \varphi+\frac{D_{T}}{T_{1}} \nabla^{2} T,
\end{gathered}
$$

where $\mathbf{V}_{D}$ is the Darcy velocity, $\mu$ and $\mu^{\prime}$ are viscosity and kinematic viscoelasticity, $\varepsilon$ is the porosity of the porous medium, $p, t$ and $\mathrm{g}$ are the pressure, time and gravity vector, $T$ is the nanofluid temperature, $\varphi$ is the nanoparticles volume fraction, $\rho$ is the density of the nanofluid, $\rho_{p}$ is the density of nanoparticles, $(\rho c)_{p}$ is the volumetric heat capacity for the nanoparticles, $(\rho c)_{f}$ is the volumetric heat capacity for the nanofluid, $(\rho c)_{m}$ is the effective heat capacity of the porous medium, $\left(k_{m}\right)$ is the effective thermal conductivity of porous media, $(\beta)$ is the volumetric thermal expansion coefficient, $\left(D_{B}\right)$ is the Brownian diffusion coefficient, $\left(D_{T}\right)$ is the thermophoresis diffusion coefficient.

On the boundaries, it is assumed that the temperature and throughflow velocity are constant. The total nanoparticle flux (sum of diffusive, thermophoretic, and connective terms) is assumed to be zero at the boundaries.

The boundary conditions are:

$$
\begin{array}{llll}
w=W_{c}, & T=T_{0}, \quad D_{B} \frac{\partial \varphi}{\partial z}-\left(\varphi-\varphi_{0}\right) W+\frac{D_{B}}{T_{\infty}} \frac{\partial T}{\partial z}=0 & \text { at } z=0 \\
w=W_{c}, & T=T_{1}, & D_{B} \frac{\partial \varphi}{\partial z}-\left(\varphi-\varphi_{0}\right) W_{c}+\frac{D_{B}}{T_{\infty}} \frac{\partial T}{\partial z}=0 & \text { at } z=1
\end{array}
$$

Defining the dimensionless variables as follows:

$$
\begin{gathered}
\left(x^{\prime}, y^{\prime}, z^{\prime}\right)=\frac{(x, y, z)}{H}, \quad \mathbf{V}\left(u^{\prime}, v^{\prime}, w^{\prime}\right)=\frac{\mathbf{V}_{D}(u, v, w) H}{\kappa}, \quad t^{\prime}=\frac{t \kappa}{\sigma H^{2}} \\
p^{\prime}=\frac{p K}{\mu \kappa}, \quad \varphi^{\prime}=\frac{\varphi-\varphi_{0}}{\varphi_{0}}, \quad T^{\prime}=\frac{T-T_{1}}{T_{0}-T_{1}}
\end{gathered}
$$

where $\kappa=\frac{k_{m}}{\left(\rho c_{p}\right)_{f}}$.

Eqs. (1)-(4) in non-dimensionless form (after dropping the primes) can be written as:

$$
\begin{gathered}
\nabla \cdot \mathbf{V}=0, \\
\frac{1}{V_{a}} \frac{d \mathbf{V}}{d t}=-\nabla \mathbf{p}-\left(1+F \frac{\partial}{\partial T}\right) \mathbf{V}-R_{m} \hat{\mathbf{k}}+R_{a} T \hat{\mathbf{k}}-R_{n} \varphi \hat{\mathbf{k}}, \\
\left(\frac{\partial}{\partial t}+\mathbf{V} \cdot \nabla\right) T=\nabla^{2} T+\frac{N_{B}}{L_{e}} \nabla \varphi \cdot \nabla T-N_{B} \varphi \mathbf{V}+\frac{N_{A} N_{B}}{L_{e}} \nabla T \cdot \nabla T \\
\frac{1}{\sigma} \frac{\partial \varphi}{\partial t}+\frac{1}{\varepsilon} \mathbf{V} \cdot \nabla \varphi=\frac{1}{L_{e}} \nabla^{2} \varphi+\frac{N_{A}}{L_{e}} \nabla^{2} T .
\end{gathered}
$$

The dimensionless parameters in Eqs. (8)-(10) namely, Prandtl number. (Pr), modified Darcy number $\left(\tilde{D}_{a}\right)$, Rayleigh number $\left(R_{a}\right)$, basic density Rayleigh number $\left(R_{m}\right)$, nanoparticle Rayleigh number $\left(R_{n}\right)$, Lewis number $\left(L_{e}\right)$, Péclet number $Q$, Modified diffusivity Ratio $\left(N_{A}\right)$, modified particle-density increment $\left(N_{B}\right)$, Vadasz 
number $(V a)$, Thermal capacity ratio $(\sigma)$, kinematics viscoelasticity $(F)$ are defined as follows:

$$
\begin{gathered}
\operatorname{Pr}=\frac{\mu}{\rho \kappa}, \quad \tilde{D}_{a}=\frac{K}{H^{2}}, \quad R_{a}=\frac{\rho g \alpha H\left(T_{0}-T_{1}\right)}{\mu \kappa}, \quad R_{m}=\frac{\left[\rho_{p} \varphi_{0}+\rho\left(1-\varphi_{0}\right)\right] g H}{\mu \kappa}, \quad R_{n}=\frac{\left\{\left(\rho_{P}-\rho\right) \varphi_{0}\right\} g H}{\mu \kappa}, \\
L_{e}=\frac{\alpha_{m}}{D_{B}}, \quad Q=\frac{H V}{k_{m}}, \quad N_{A}=\frac{D_{T}\left(T_{0}-T_{1}\right)}{D_{B} T_{1} \varphi_{0}}, \quad N_{B}=\frac{(\rho c)_{p} \varphi_{0}}{(\rho c)_{f}}, \quad V a=\frac{\varepsilon \operatorname{Pr}}{D a}, \quad \sigma=\frac{\left(\rho c_{p}\right)_{m}}{\left(\rho c_{p}\right)_{f}}, \quad F=\frac{\mu^{\prime} K}{\mu \sigma H^{2}} .
\end{gathered}
$$

The dimensionless form of boundary conditions are written as:

\subsection{Basic solutions}

$$
\begin{array}{llll}
w=Q, & T=1, & \frac{\partial \varphi}{\partial z}-Q L_{e} \varphi+N_{A} \frac{\partial T}{\partial z}=0 & \text { at } z=0 \\
w=Q, & T=0, & \frac{\partial \varphi}{\partial z}-Q L_{e} \varphi+N_{A} \frac{\partial T}{\partial z}=0 & \text { at } z=1 .
\end{array}
$$

The basic state of nanofluid is assumed to be time independent and is described by:

$$
\mathbf{V}=\mathbf{V}_{\mathbf{b}}=(0,0, Q), \quad p=p_{b}(z), \quad T=T_{b}(z), \varphi=\varphi_{b}(z) .
$$

Then, Eqs. (8)-(10) are simplified as:

$$
\begin{gathered}
\frac{d^{2} T_{b}}{d z^{2}}+\frac{N_{B}}{L_{e}} \frac{d \varphi_{b}}{d z} \frac{d T_{b}}{d z}+\frac{N_{A} N_{B}}{L_{e}}\left(\frac{d T_{b}}{d z}\right)^{2}-Q_{v} \frac{d T_{b}}{d z}-Q_{v} N_{B} \varphi_{b} \frac{d T_{b}}{d z}=0, \\
\frac{d^{2} \varphi_{b}}{d z^{2}}+N_{A} \frac{d^{2} T_{b}}{d z^{2}}-Q_{v} L_{e} \frac{d \varphi_{b}}{d z}=0 .
\end{gathered}
$$

Solving Eq. (15) and Eq. (16) with boundary condition gives:

$$
\begin{gathered}
T_{b}=\frac{e^{Q_{v}}-e^{Q_{v} z}}{e^{Q_{v}}-1}, \\
\varphi_{b}=\frac{\left(1-L_{e}-N_{A}\right)\left(e^{Q_{v} L_{e} z}-1\right)}{\left(1-L_{e}\right)\left(e^{Q_{v} L_{e}}-1\right)}+\frac{N_{A}\left(e^{Q_{v} z}-1\right)}{\left(1-L_{e}\right)\left(e^{Q_{v}}-1\right)} .
\end{gathered}
$$

We differentiate the $T_{b}, \varphi_{b}$ with respect to $z$ :

$$
\begin{gathered}
\frac{d T_{b}}{d z}=\frac{Q e^{Q z}}{\left(1-e^{Q}\right)} \\
\frac{d \varphi_{b}}{d z}=\frac{Q L_{e}\left(\varepsilon-L_{e}-N_{A}\right) e^{\frac{Q L_{e} z}{\varepsilon}}}{\left(1-L_{e}\right) \varepsilon\left(e^{\frac{Q L_{e}}{\varepsilon}}-1\right)}+\frac{\varepsilon N_{A} Q e^{Q z}}{\left(\varepsilon-L_{e}\right)\left(e^{Q}-1\right)} .
\end{gathered}
$$

Using the limit $Q \rightarrow 0$, the basic solution becomes $T_{b}=1-z, \varphi_{b}=z$; this is same solution as obtained by [11].

\subsection{Perturbed solutions}

For perturbations of small disturbance onto the base fluid, we assume that:

$$
\mathbf{V}=\mathbf{V}_{b}+\mathbf{V}^{\prime}, \quad T=T_{b}+T^{\prime}, \quad \varphi=\varphi_{b}+\varphi^{\prime}, \quad p=p_{b}+p^{\prime} .
$$

Substituting the perturbed solutions in Eqs. (7)-(10) and Eq. (12), we get:

$$
\begin{gathered}
\nabla \cdot \mathbf{V}^{\prime}=0 \\
\frac{1}{V_{a}} \frac{\partial \mathbf{V}^{\prime}}{\partial t}+w^{\prime}=-\frac{\partial p^{\prime}}{\partial z}-\left(1+F \frac{\partial}{\partial t}\right)+R_{a} T^{\prime} \hat{\mathbf{k}}-R_{n} \varphi^{\prime} \hat{\mathbf{k}}^{\prime}, \\
\frac{\partial T^{\prime}}{\partial t}+\frac{d T_{b}}{d z} w^{\prime}+Q \frac{\partial T^{\prime}}{\partial z}=\nabla^{2} T^{\prime}+\frac{N_{B}}{L_{e}}\left(\frac{d \varphi_{b}}{d z} \frac{\partial T^{\prime}}{\partial z}-\frac{d T_{b}}{d z} \frac{\partial \varphi^{\prime}}{\partial z}\right)+\frac{2 N_{A} N_{B}}{L e} \frac{d T_{b}}{d z} \frac{\partial T^{\prime}}{\partial z} \\
-N_{B}\left(Q \frac{d T_{b}}{d z} \varphi^{\prime}+\varphi_{b} \frac{d T^{\prime}}{d z} w^{\prime}+Q \varphi_{b} \frac{\partial T^{\prime}}{\partial z}\right), \\
\frac{\partial \varphi^{\prime}}{\partial t}+Q \frac{\partial \varphi^{\prime}}{\partial z}+\frac{d \varphi_{b}}{d z} w^{\prime}=\frac{1}{L_{e}} \nabla^{2} \varphi^{\prime}+\frac{N_{A}}{L_{e}} \nabla^{2} T^{\prime} .
\end{gathered}
$$

Eq. (22) is operated with $\hat{\mathbf{k}} \cdot$ curl curl and the use is made of the identity curl curl $=$ graddiv $-\nabla^{2}$ together with Eq. (21). As a result of this procedure, pressure is eliminated from Eq. (22) and we get:

$$
\frac{1}{\operatorname{Pr}} \frac{\partial}{\partial t} \nabla^{2} w^{\prime}+\left(1+M+F \frac{\partial}{\partial T}\right) \nabla^{2} w^{\prime}=R_{a} \nabla_{H}^{2} T^{\prime}-R_{n} \nabla_{H}^{2} \phi^{\prime}
$$


In Eq. (25) $\nabla_{H}^{2}$ is the 2-D Laplacian operator in the horizontal plane.

Analyzing the disturbance into the normal modes, perturbation equations are introduced as the following form:

$$
\left[w^{\prime}, T^{\prime}, \varphi^{\prime}\right]=[W(z), \Theta(z), \Phi(z)] f(x, y) \exp (s t)
$$

Using the normal mode analysis, the following equations for the amplitudes $\Theta, \Phi, W$ are obtained:

$$
\begin{aligned}
&\left(D^{2}-a^{2}\right)\left(1+\frac{s}{V_{a}}+s F\right) W+R_{a} a^{2} \Theta-R_{n} a^{2} \Phi=0, \\
&\left(-1+N_{B} Q\right) \frac{d T_{b}}{d z} W+\left(D^{2}+\frac{N_{B}}{L_{e}} \frac{d \varphi_{b}}{d z} D+\frac{2 N_{A} N_{B}}{L_{e}} \frac{d T_{b}}{d z} D-N_{B} Q \varphi_{b} D-Q_{v} D-a^{2}-s\right) \Theta \\
&+\left(\frac{N_{B}}{L_{e}} \frac{d T_{b}}{d z} D-N_{B} Q \frac{d T_{b}}{d z}\right) \Phi=0, \\
& \frac{d \varphi_{b}}{d z} W-\frac{N_{A}}{L_{e}}\left(D^{2}-a^{2}\right) \Theta-\frac{1}{L_{e}}\left(\left(D^{2}-a^{2}\right)-Q_{v} D-\frac{s}{\sigma} \Phi\right)=0,
\end{aligned}
$$

with boundary conditions as:

$$
W=0, \quad \Theta=0, \quad D \Phi-\frac{Q L_{e}}{\varepsilon} \Phi+N_{A} D \Theta=0, \quad \text { at } z=0,1 .
$$

Here, $a$ is a dimensionless wave number, and $s=i \omega$ is a complex growth factor, where $\omega$ is a dimensionless frequency (it is a real number).

Single-term Galerkin-type weighted residuals method is used to obtain approximate solutions to the system of Eqs. (27)-(29). Accordingly $\Theta, W, \Phi$ (satisfying the boundary conditions exactly) are chosen as:

$$
\Theta_{1}=z-z^{2}, \quad W_{1}=z(1-z)^{2}, \quad \Phi_{1}=\frac{-N_{A}\left(Q L_{e}-2-2 Q L_{e} z\right)}{Q L_{e}^{2}} .
$$

We substitute this minimal polynomial into Eqs. (27)-(29) and using the Orthogonality of trial functions, we obtain a system of 3 linear algebraic equations in the 3 unknowns. We get:

$$
\left[\begin{array}{clc}
G 1 & \frac{R_{a} a^{2}}{140} & \frac{R_{n} N_{A} a^{2}}{15 \eta^{2}} \\
\left(-1+N_{B} Q\right) A_{T W} & G 2 & \frac{N_{B}}{L_{e}} B_{T \Phi}-N_{B} Q A_{T \Phi} \\
A_{\varphi W} & -\frac{N_{A}^{2}\left(12+a^{2}\right)}{3 L_{e} \eta^{2}} & \frac{N_{A}^{2} a^{2}\left(12+\eta^{2}\right)-3-s\left(12+\eta^{2}\right)}{3 L_{e} \eta^{4}}
\end{array}\right]\left[\begin{array}{c}
W \\
\Theta \\
\Phi
\end{array}\right]=\left[\begin{array}{l}
0 \\
0 \\
0
\end{array}\right]
$$

where

$$
\begin{gathered}
G 1=\left(\frac{S}{V a}+1\right)\left(504+24 a^{2}+a^{4}+\tilde{D}_{a}\left(12+a^{2}\right)\right) \\
G 2=\frac{-\left(10+a^{2}\right)}{30}+\frac{N_{B}}{L_{e}} B \varphi \Theta+\frac{2 N_{A} N_{B}}{L_{e}} B_{T \Theta}-\frac{s}{30}, \\
A_{T W}=\int_{0}^{1}\left(\frac{d T_{b}}{d z} W\right) \Theta d z=\frac{\left.6\left(120+60 Q+12 Q^{2}+Q^{3}\right)+e^{Q}(-120+Q(60+(-12+Q) Q))\right)}{Q^{6}\left(1-e^{Q}\right)}, \\
B_{\varphi \Theta}=\int_{0}^{1}\left(\frac{d \varphi_{b}}{d z} D \Theta\right) \Theta d z=-\frac{\eta^{2}+6 \eta+12-e^{\eta}\left(\eta^{2}-6 \eta+12\right)\left(Q-\eta-N_{A} Q\right)}{\eta^{3}\left(1-e^{\eta}\right)(Q-\eta)} \\
B_{T \Theta}=\int_{0}^{1}\left(\frac{d T_{b}}{d z} D \Theta\right) \Theta d z=\frac{N^{2}+6 Q+12-e^{Q}\left(Q^{2}-6 Q+12\right)}{Q^{2}\left(1-e^{Q}\right)(Q-\eta)} \\
A_{\varphi \Theta}=\int_{0}^{1}\left(\varphi_{b} D \Theta\right) \Theta d z=\frac{\eta^{2}+6 \eta+12-e^{\eta}\left(\eta^{2}-6 \eta+12\right)\left(Q-\eta-N_{A} Q\right)}{\eta^{4}\left(1-e^{\eta}\right)(Q-\eta)} \\
+\frac{N_{A}\left(Q^{2}+6 Q+12-e^{Q}\left(Q^{2}-6 Q+12\right)\right)}{Q^{3}\left(1-e^{Q}\right)(Q-\eta)},
\end{gathered}
$$




$$
\begin{gathered}
B_{T \Phi}=\int_{0}^{1}\left(\frac{d T_{b}}{d z} D \Phi\right) \Theta d z=\frac{2 \eta\left(2+e^{Q}(-2+Q)+Q\right)}{Q^{2}\left(1-e^{Q}\right)}, \\
A_{T \Phi}=\int_{0}^{1}\left(\frac{d T_{b}}{d z} \Phi\right) \Theta d z=\frac{-2 Q\left(2+e^{Q}(-2+Q)+Q\right)+\eta\left(12+6 Q+Q^{2}-e^{Q}\left(12-6 Q+Q^{2}\right)\right)}{Q^{3}\left(1-e^{Q}\right)}, \\
A_{\varphi \Phi}=\int_{0}^{1}\left(\frac{d \varphi_{b}}{d z} W\right) \Phi d z=\frac{2\left(Q-\eta-N_{A} Q\right)(\eta-2(1+\eta z))\left(-12-\eta(6+\eta)+e^{\eta}(12+(-6+\eta) \eta)\right)}{\eta^{4}(Q-\eta)\left(e^{\eta}-1\right)} \\
+\frac{2 N_{A}(\eta-2(1+\eta z))\left(-12-Q(6+Q)+e^{Q}(12+(-6+Q) Q)\right)}{Q^{3}(Q-\eta)\left(e^{Q}-1\right)}, \\
\eta=\frac{Q L_{e}}{\varepsilon} .
\end{gathered}
$$

For all considered set of boundary conditions, oscillatory convection cannot be occurring. In the absence of oscillatory convection $(s=0)$, we get the following expression for $R_{a}$ :

$$
R_{a}=\frac{14\left(\left(504+24 a^{2}+a^{4}+\tilde{D}_{a}\left(12+a^{2}\right)\right) \alpha-N_{A} R_{n} \beta\right)}{\gamma}
$$

where:

$$
\begin{aligned}
& \begin{array}{l}
\alpha= \\
\frac{30 \eta^{2} N_{A}^{2} N_{B}\left(12+a^{2}\right)\left(B_{T \Phi}-Q A_{T \Theta} L_{e}\right)+A_{\varphi W} \eta^{2}\left(N_{a}^{2} a^{2}\left(12+\eta^{2}\right)-3\right)\left(N_{B}\left(B_{\Phi \Theta}+2 B_{T \Theta} N_{A}\right)-\left(10+a^{2}\right) L_{e}\right)}{9 a^{2} L_{e}^{2} \eta^{4}} \\
\beta=\frac{A_{\varphi W} \eta^{2}\left(30 N_{B}\left(B_{\Phi \Theta}+2 B_{T \Theta} N_{A}\right)-\left(10+a^{2}\right) L_{e}\right) A_{\varphi W} \eta^{2}+10 N_{A}^{2} A_{T W}\left(-1+N_{B} Q\right)\left(12+a^{2}\right)}{45 L_{e} \eta^{4}} \\
\gamma=\frac{3 L_{e} \eta^{4} N_{B}\left(B_{T \Phi}-Q A_{T \Theta} L_{e}\right)-A_{T W}\left(N_{a}^{2} a^{2}\left(12+\eta^{2}\right)-3\right)\left(-1+N_{B} Q\right)}{3 L_{e} \eta^{4}}
\end{array}
\end{aligned}
$$

The Rayleigh number $\left(R_{a}\right)$ is a function of $a \varepsilon, \tilde{D}_{a}, Q, L_{e}, N_{A}$, a $R_{n}$, and $N_{B}$ and from Eq. (32), it is also clear that the Rayleigh number $\left(R_{a}\right)$ is independent of viscoelastic parameters $\left(V_{a}, F, \sigma\right)$. Thus, the effect of throughflow in Rivlin-Ericksin elastico-viscous nanofluid is independent of viscoelastic parameters. To simplify the above expression, we have assumed that $Q \ll 1$, so that first order Galerkin approximation leads to a useful result:

$$
R_{a}=\frac{140(G 3)\left(504+24 a^{2}+a^{4}+\tilde{D}_{a}\left(12+a^{2}\right)\right)-\left(105\left(12+a^{2}\right)\left(1+N_{B} Q\right) N_{A}-98(G 3) L_{e}\right) R_{n}}{135 a^{2}\left(1+N_{B} Q\right)},
$$

where $G 3=10+a^{2}-\frac{N_{A} N_{B} Q}{L_{e}}$.

In Eq. (33), for the admissible range of parameters the Rayleigh number decreases as a nanoparticle Rayleigh number increases. The corresponding critical wave number can be obtained from Eq. (33), which in the presence of throughflow, critical wave number is a function of the P'eclet number, modified Darcy number as well as nanofluid parameters.

In the absence of vertical throughflow, Eq. (33) reduces to the following form:

$$
R_{a}=\frac{140\left(10+a^{2}\right)\left(504+24 a^{2}+a^{4}+\tilde{D}_{a}\left(12+a^{2}\right)\right)-105\left(12+a^{2}\right) N_{A} R_{n}-98\left(10+a^{2}\right) L_{e} R_{n}}{135 a^{2}} .
$$

In the absence of vertical throughflow $(Q=0)$ the Rayleigh number is not a function of the modified particle density increment $\left(N_{B}\right)$. 


\section{Results and discussions}

To study the effect of various parameters on the stability, Eq. (33) is analyzed analytically and numerically. According to data given by $[1,11]$, the following threshold values for alumina/water nanofluids of $1-100 \mathrm{~nm}$ are utilized: $L_{e}=500, Q=0.5, R_{n}=0.1, N_{A}=5, N_{b}=0.01$. Fig. 1 shows the plot of crtical Rayleigh number $\left(R_{a, c}\right)$ versus nanoparticle Rayleigh number $R_{n}$ for vertical throughflow $Q=0.1,0.3,0.7,1$. It can be seen that $R_{n}$ destablizes the onset of convection. Fig. 1 also shows that $R_{a, c}$ increases with increasing value of $Q$. Vertical through flow delays the onset of nanofluid convection and this effect is larger for higher nanoparticle Rayleigh number values.

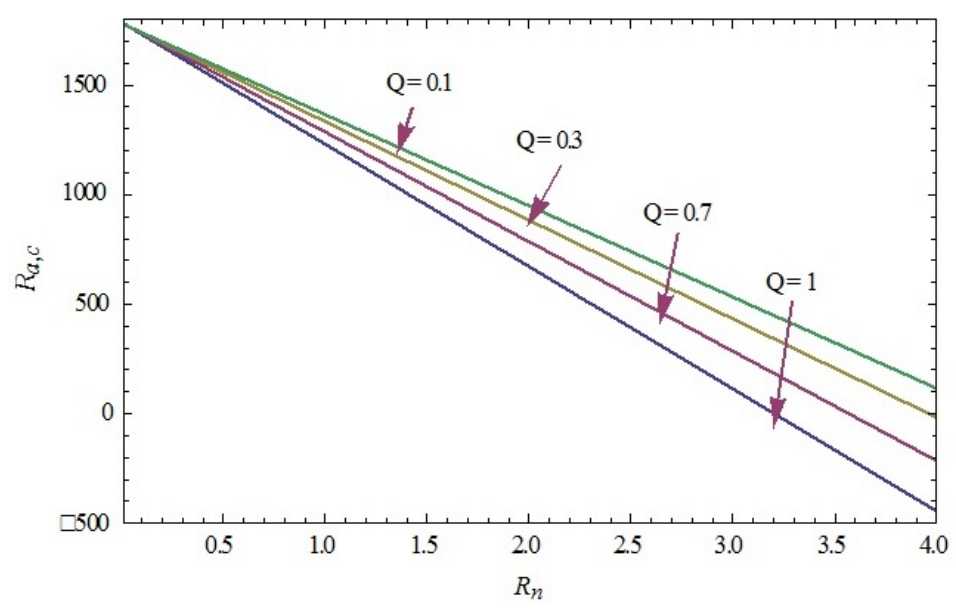

FIG. 1. Variation of $R_{a, c}$ with $R_{n}$ for different values of $Q$

Figure 2 shows that the modified particle density increment $\left(N_{B}\right)$ has no significant effect on the stability of Rivlin-Ericksin elastico-viscous nanofluid.

Figure 3 shows the variation of Rayleigh number $R_{a}$ when $L_{e}=500,700$ and $N_{A}=1.5$ respectively. It is seen that as the value of the Lewis number $\left(L_{e}\right)$ increases, the Rayleigh number decreases. Thus, the Lewis number promotes nanofluid convection. The modified diffusivity ratio has a destabilizing effect on the onset of convection. This is because thermophoresis pushes the lighter nanoparticles upwards, which enhances the destabilizing effects on particle distributions.

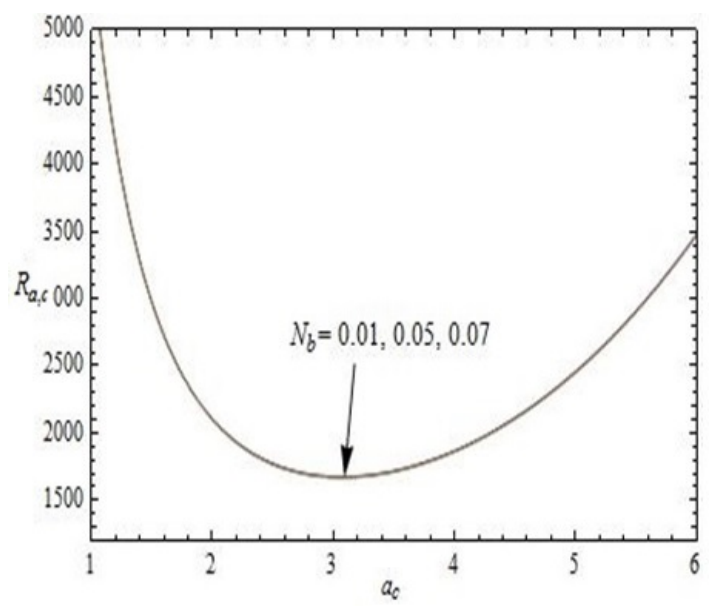

FIG. 2. Variation of $R_{a, c}$ with $a_{c}$ for different values of $N_{b}$

\section{Conclusions}

In this paper, we invistigate the effect of vertical throughflow in Rivlin-Ericskin elastico-viscous nanofluid for Rigid-rigid boundaries in a non-Darcy porous medium. It was observed that when using the conective term 


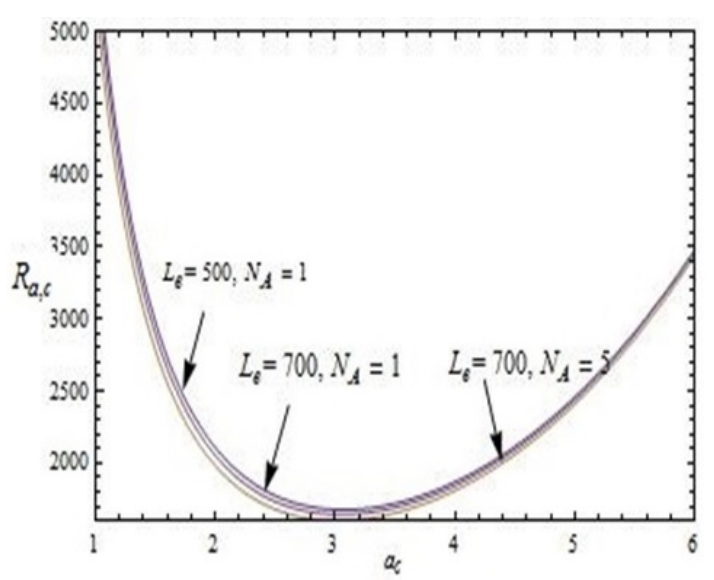

FIG. 3. Variation of $R_{a, c}$ with $a_{c}$ for different values of $N_{b}, L_{e}$

of nanoparticle flux at the boundary, the Rayleigh number was independent of thermal capacity ratio, kinematics viscoelasticity parameter and Vadsaz number. The critical wave number was a function of the nanofluid parameters as well as Péclet number. Porosity, Lewis number, and nanoparticle Rayleigh number promotes instablity while the modified particle-density increament had no significant effect on the stablity. Vertical through flow delays the onset of nanofluid convection and this effect is larger for higher nanoparticle Rayleigh number values. New boundary condtions in terms of zero nanoparticle flux (the sum of the diffusive, thermophoretic, and connective terms) is more favourable for convection; however there is no drastic change in the magnitude of Rayleigh number.

\section{References}

[1] Rivlin R., Ericksen J. Stress-Deformation Relations for Isotropic Materials. J. Ration Mec.h Anal., 1955, 4(2), P. 323-425.

[2] Rudraiah N., Kaloni P.N., Radhadevi P.V. Oscillatory convection in a viscoelastic fluid through a porous layer heated from below. Rheologica Acta., 1989, 28(1), P. 48-53.

[3] Kim M.C., Lee S.B., Chung B.J. Thermal instability of viscoelastic fluids in porous media. Int. J. Heat Mass Transf., 2003, 46, P. 50655072.

[4] Sharma R.C., Kumar P. Effect of Rotation on Thermal Instability in Rivlin-Ericksen elastico-viscous Fluid. Zeitschrift für Naturforschung A., 1996, 51(7), P. 821-824.

[5] Sharma R.C., Kumar P. Thermal Instability in Rivlin-Ericksen elastico-viscous Fluid in Hydromagnetic. Z. Naturforsch., 1997, 52a, P. 369-371.

[6] Sheu L.J. Thermal Instability in a Porous Medium Layer Saturated with a Visco-Elastic Nanofluid. Transp. Porous Med., 2011, 88, P. 461-477.

[7] Sheu L.J. Linear Stability of Convection in a Visco Elastic Nanofluid Layer. World Acad. Sci. Eng. Technol., 2011, 58, P. $289-295$.

[8] Choi S. Enhancing Thermal Conductivity of Fluids with Nanoparticles in Developments and Applications of Non-Newtonian Flows. ASME, 1995, 66, P. 99-105.

[9] Buongiorno J. Connective Transport in nanofluids. J. Heat Transf., 2006, 128, P. 240-250.

[10] Nield D.A., Kuznetsov A.V. Thermal instability in a porous medium layer saturated by a nanofluid. Int .J. Heat Mass Transf., 2009, 52, P. 5796-5801.

[11] Nield D.A., Kuznetsov A.V. The onset of convection in a horizontal layer of finite depth. Eur. J. Mech. B/Fluids, 2010, 29, P. 217-223.

[12] Homsy G.M., Sherwood A.E. Convective instabilities in porous media with throughflow. AIChE J., 1976, 22, P. 168-174.

[13] Jones M.C., Persichetti J.M. Convective instability in packed beds with throughflow. AIChE J., 1986, 32, P. $1555-1557$.

[14] Nield D.A. Convective instability in porous media with throughflow. AIChE J., 1987, 33, P.1222-1224.

[15] Barletta A., di Schio E. R., Storesletten L. Convective roll instabilities of vertical throughflow with viscous dissipation in a horizontal porous layer. Transp. Porous Med., 1986, 81, P. 461-77.

[16] Nield D.A., Kuznetsov A.V. The effect of vertical through flow on thermal instability in a porous medium layer saturated by a nanofluid. Transp. Porous Med., 2011, 87, P. 765-775.

[17] Nield D.A., Kuznetsov A.V. The onset of convection in a layered porous medium with vertical throughflow. Transp. Porous Med. 2013, 98, P. 363-376.

[18] Nield D.A., Kuznetsov A.V. The effect of vertical throughflow on thermal instability in a porous medium layer saturated by a nanofluid: a revised model. ASME J. Heat Transf., 2015, 137, P. 052601.

[19] Khalili A., Shivakumara I.S. Onset of convection in a porous layer with net throughflow and internal heat generation. Phys Fluids, 1998, 10, P. 315.

[20] Nield D.A., Kuznetsov A.V. The effects of combined horizontal and vertical heterogeneity on the onset of convection in a porous medium with horizontal throughflow. Int. J. Heat Mass Transf., 2010, 54, P. 5595-5601. 